\title{
Meta-análisis del efecto en el rendimiento académico de los Smartphones como herramienta educativa en educación superior (2016-2020)
}

\section{Meta-analysis of the effect on academic performance of smartphones as an educational tool in higher education (2016-2020)}

\author{
Belinda UxaCH Molina ${ }^{2}$ \\ Universidad Complutense de Madrid, España \\ buxach@ucm.es \\ https://orcid.org/0000-0001-8613-4621
}

\begin{abstract}
Resumen:
La presente investigación pretende aunar los esfuerzos de distintos investigadores realizados en los últimos 5 años (20162020) sobre el análisis del efecto producido por los Smartphones en la educación reglada, concretamente en el ámbito universitario. En relación con la metodología seguida se tomó como referencia las fases recomendadas por Meca (2010). Los estudios incluidos en este meta-análisis han sido seleccionados de entre los que se obtuvieron como respuesta en la búsqueda de las siguientes bases de datos: Scopus, WoS, Eric y Dialnet, y cumplían con los criterios determinados a priori basados en las necesidades del estudio. Finalmente
\end{abstract}

\begin{abstract}
:
This study aims to bring together the efforts made by different researchers over the last 5 years (2016-2020) regarding the analysis of the effect produced by Smartphones in formal education, specifically in university settings. In relation to the methodology followed, the phases recommended by Meca (2010) were taken as a reference. The studies included in this meta-analysis were selected through a search in the following databases: Scopus, WoS, Eric and Dialnet, and met the criteria determined a priori based on the needs of the study. The final selection included 25 articles, after a total of 2286 articles were subjected to rigorous analysis. During
\end{abstract}

1 Como referenciar este artículo (How to reference this article):

Uxach Molina, B. (2021 Meta-análisis del efecto en el rendimiento académico de los Smartphones como herramienta educativa en educación superior (2016-2020). Educatio Siglo XXI, 39(3), 209-234. https://doi.org/10.6018/educatio.431031

2 Dirección para correspondencia (Correspondence address):

Belinda Uxach Molina. Departamento de Estudios Educativos. Facultad de Educación. Centro de Formación del Profesorado. Edificio La Almudena. C/ Rector Royo Villanova, s/n. Ciudad Universitaria. 28040 Madrid (España). 
Uxach Molina, B. (2021). Meta-análisis del efecto en el rendimiento académico de los Smartphones como herramienta educativa en educación superior (2016-2020). Educatio Siglo XXI, 39(3),

209-234.

25 artículos configuraron la población de este meta-análisis tras haber sido sometidos un total de 2286 a riguroso análisis. Durante la investigación se consideró necesario analizar la influencia del tiempo, al que estuvo cada grupo expuesto a la metodología m-learning y las posibles diferencias entre estudios sobre idiomas $u$ otros ámbitos en la magnitud del efecto. Los resultados obtenidos, en cuanto a la magnitud del efecto y los análisis realizados, muestran una clara influencia positiva de la metodología m-learning en el rendimiento académico de los alumnos. Por tanto, se considera congruente la implementación de la metodología m-learning en las aulas universitarias y la profundización en el estudio de su efecto explorando otros niveles y áreas de conocimiento.

\section{Palabras clave:}

Aprendizaje activo; método de aprendizaje; autoaprendizaje; tecnología educacional. the investigation, the authors considered necessary to analyze (i) the influence of time of exposure of each group to the mlearning methodology and (ii) the possible differences between studies on languages or other aspects in the magnitude of the effect. In terms of the magnitude of the effect and the analyses conducted, the results show a clear positive influence of the m-learning methodology on students' academic performance. Therefore, implementing m-learning methodology in university classrooms and to study its effect in depth by exploring other levels and areas of knowledge is considered congruent.

\section{Keywords:}

Active learning; learning method; selflearning; educational technology.

\section{Résumé:}

Cette recherche vise à rassembler les efforts de différents chercheurs menés au cours des 5 dernières années (2016-2020) sur l'analyse de l'effet produit par les Smartphones dans l'éducation formelle, spécifiquement dans l'environnement universitaire.

En ce qui concerne la méthodologie suivie, les phases recommandées par Meca (2010) ont été prises comme référence. Les études incluses dans cette méta-analyse ont été sélectionnées parmi celles obtenues en réponse à la recherche dans les bases de données suivantes : Scopus, WoS, Eric et Dialnet, et répondaient aux critères déterminés a priori en fonction des besoins de l'étude. Finalement, 25 articles ont constitué la population de cette méta-analyse après que 2286 au total aient été soumis à une analyse rigoureuse. Au cours de la recherche, il a été jugé nécessaire d'analyser l'influence du temps pendant lequel chaque groupe a été exposé à la méthodologie m-learning et les éventuelles différences entre les études sur les langues ou d'autres domaines sur l'ampleur de l'effet. Les résultats obtenus, en termes de magnitude de l'effet et des analyses effectuées, montrent une influence positive claire de la méthodologie m-learning sur les performances académiques des étudiants. Par conséquent, la mise en œuvre de la méthodologie mlearning dans les salles de classe universitaires et l'étude plus approfondie de son effet explorant d'autres niveaux et domaines de connaissance sont considérées comme congruentes.

Mots-clés:

Apprentissage actif; méthode d'apprentissage; auto-apprentissage; technologie educative.

Fecha de recepción: 07-06-2020

Fecha de aceptación: 18-01-2021 


\section{Introducción}

La implementación de la metodología m-learning, aprendizaje mediado por Smartphones en este caso, en el ámbito universitario origina numerosas investigaciones sobre el efecto producido en el rendimiento académico del alumnado. Este meta-análisis trata de analizar la magnitud conjunta de este efecto tomando como base las publicaciones editadas en los últimos 5 años (2016-2020) en diferentes revistas. Scopus, WoS, Eric y Dialnet son las bases de datos escogidas para la exploración y selección de los artículos. Un total de 2286 investigaciones fueron revisadas y codificadas para ser sometidas a riguroso análisis, de las cuales, 25 cumplieron con los criterios definidos en el diseño y por tanto configuraron la muestra poblacional de esta investigación.

Los criterios de selección definidos a priori se basaron en otras investigaciones de características similares (Díaz, Reche y Rodríguez, 2018 y Wu, et al. 2012) y en las recomendaciones de otros autores sobre la realización de revisiones sistemáticas y meta-análisis (Urrutia, y Bonfill, 2010).

La presente investigación sigue una estructura cuatripartita además de la presente introducción y las imprescindibles conclusiones. En primer lugar, se presentan los objetivos, generales y específicos que se pretenden alcanzar. En segundo lugar, se describirá detalladamente la metodología seguida durante el proceso donde se describirán los criterios de selección de artículos y herramientas utilizadas. Seguidamente, se expondrán los resultados obtenidos tras el trabajo de análisis. Finalmente, serán comentados estos resultados relacionándolos con los objetivos del trabajo.

El propósito principal de esta investigación es cuantificar la magnitud del efecto conjunto en el rendimiento académico de alumnos universitarios tras la experimentación de la metodología m-learning utilizando como base las investigaciones publicadas en los últimos 5 años. La hipótesis se articula en torno a que este efecto es positivo y por tanto la utilización de los Smartphones como herramienta educativa mejora el aprendizaje y, por lo tanto, el rendimiento académico. 


\section{Estado de la cuestión}

El desarrollo tecnológico ha permitido la conversión de los Smartphones en herramientas suficientemente potentes como para competir con los ordenadores proporcionando, además, versatilidad, movilidad, autonomía y mayor facilidad de adquisición debido a su coste inferior. Hoy en día la tecnología móvil está tan extendida que forma parte de la vida de adolescentes y adultos. La posesión y uso de Smartphones roza el $100 \%$ de la población mayor de edad en España, según el INE, en 2019 la disposición de terminales aumentó a partir de los 10 años llegando al 93,8\% a los 15 años. Según esta misma encuesta, el 95,0\% de la población de 16 a 74 años (31,7 millones de usuarios) utiliza el móvil para acceder a internet fuera de la vivienda habitual o el lugar de trabajo. Los servicios de mensajería instantánea para comunicarse con familiares y amigos son los más utilizados por los encuestados (93,8\%), seguido de la búsqueda de servicios $(80,3 \%$ ) y la revisión de correos electrónicos (79,6\%) (INE, 2019). Esta información estadística demuestra la extendida presencia y familiaridad con los terminales móviles existente dibujando un escenario favorable para la implementación del m-learning.

Las tarifas planas y asequibles, los centros públicos con wifi de libre acceso (bibliotecas, bares, autobuses...) contribuyen a mantener a la sociedad conectada en todo momento. Obtenemos información y utilizamos numerosas aplicaciones de opinión donde realizamos continuamente juicios críticos sobre informaciones recibidas, acontecimientos, experiencias... Sin embargo, esta tecnología está incorporándose en las esferas educativas a un ritmo infinitamente inferior al que lo ha hecho en nuestras vidas y en consecuencia los alumnos experimentan una disonancia entre sus vidas dentro y fuera del aula.

Se entiende el m-learning [...] como una metodología de enseñanza y aprendizaje que se vale del uso de pequeños dispositivos móviles, tales como teléfonos móviles, PDA, tabletas, PocketPC, iPod y todo otro dispositivo de mano que tenga alguna forma de conectividad inalámbrica. [...] hace referencia al aprendizaje apoyado en la tecnología y que se puede realizar en cualquier momento y desde cualquier lugar.

(Santiago y Trabaldo, 2015, p.5). 
La defensa que numerosos autores realizan de la implementación en las aulas de metodologías basadas en el m-learning, utilizando dispositivos móviles como herramienta educativa, se articula en torno a tres conceptos claves. La motivación, la comunidad educativa y la significación del aprendizaje ubicuo. Otros autores (Brazuelo y Gallego, 2011 y Fernández, 2016) destacan también de esta modalidad educativa la facilidad de construcción de conocimiento, la resolución de problemas de aprendizaje (en relación con la inclusión educativa), y el desarrollo autónomo y ubicuo de destrezas gracias a la medicación de dispositivos móviles. A lo que Burbules (2012) añade que proporciona la oportunidad de relacionar las metas de aprendizaje con contextos y propósitos más allá del salón de clases.

En cuanto a la motivación, la literatura confirma la influencia de los fenómenos afectivos, concretamente las motivaciones, los sentimientos y las emociones que se generan en los contextos escolares, en el impulso del aprendizaje y el rendimiento (Pérez, 2009). Entre las atribuciones de los docentes se encuentra la de adaptar los modelos educativos a las necesidades del alumnado con el fin de mantener la motivación de estos durante todo el proceso de aprendizaje. Uno de los puntos esenciales para ello es captar su atención y mantenerla conectando los conocimientos y destrezas que se esperan que aprenden con sus intereses personales y su vida cotidiana (García y Álvarez, 2007). Considerando entonces que los alumnos están en constante contacto con los dispositivos móviles (Smartphones, tabletas...) una forma de promover el interés podría ser la introducción de estas herramientas en el aula y servirnos de ellas para resolver problemas y construir conocimiento en comunidad. La prohibición de estos dispositivos en las aulas no hace más que romper la conexión y alejar las experiencias educativas del resto de la vida. Sin embargo, su inclusión en ellas extendería el contexto físico y temporal del aprendizaje fuera del tiempo y espacio delimitado por el centro educativo gracias a la ubicuidad de los terminales (Grund y González, 2017).

Así mismo, numerosos autores afirman que es a través del aprendizaje significativo el alumno construye el conocimiento favoreciendo su crecimiento personal. De acuerdo con la teoría constructivista, el alumno selecciona, organiza y transforma la información que puede recibir de distintas fuentes y establece relaciones con sus conocimientos previos para construir el conocimiento (Díaz y Hernández, 2015). Esto 
Uxach Molina, B. (2021). Meta-análisis del efecto en el rendimiento académico de los Smartphones como herramienta educativa en educación superior (2016-2020). Educatio Sig/o XXI, 39(3), 209-234.

define al docente como un guía encargado de proporcionar al alumno los apoyos necesarios para acompañarle en ese proceso. Este guía debe servirse de todas aquellas herramientas que le sean posibles para apoyar el proceso enseñanza-aprendizaje y los terminales móviles constituyen una herramienta muy completa para ello. Entre las características de estos aparatos que algunos autores asocian a los procesos educativos se encuentran:

- La posibilidad de acceso rápido y actualizado a la información desde cualquier lugar.

- El uso de aplicaciones útiles para la comunicación y representación que invitan a la reflexión y crítica de los contenidos.

- La adaptabilidad de servicios, aplicaciones e interfaces a las necesidades del usuario (relacionado con la educación inclusiva y las distintas capacidades y características del alumnado por motivos físicos, psicológicos o sociales).

- La existencia de aplicaciones de evaluación y seguimiento del proceso basadas en juegos y actividades atractivas vinculadas con los intereses y el entorno social del alumnado (Realidad Aumentada, códigos QR, WhatsApp, Kahoot...) que captan la atención y ayudan a mantenerla a lo largo de todo el proceso.

- Y finalmente, la capacidad de crear comunidades de aprendizaje conectadas con centros de otros lugares y culturas distintas promoviendo así, por un lado, su conocimiento y aceptación y por otro el desarrollo de sentimiento de pertenencia y relación de la cultura propia con los contenidos académicos.

Inmersos en esta nueva metodología que posibilita la deslocalización el proceso de enseñanza-aprendizaje muchas son las investigaciones centradas en el estudio de las percepciones de los participantes (Chrifi et al., 2019; Jabali et al., 2019; Khan et al., 2019; Klimova y Polakova, 2020 y Wang y Hsu, 2020). El alumnado destaca de la metodología mlearning grandes potencialidades para mejorar los procesos pedagógicos (Contreras, 2010), poniendo el acento en la facilidad de uso de los Smartphones y en la utilidad percibida tras su experiencia (López y Silva, 2016). Demuestran una alta aceptación entre el colectivo universitario que define los Smartphones como herramientas innovadoras y originales contextualizadas capaces de ampliar el mero uso informal al uso educativo (Martín-Gutiérrez et al., 2010). Entre las funcionalidades más desta- 
cadas de los Smartphones en las distintas publicaciones se encuentran la percepción de estos como un medio para la comunicación; un medio para la gestión de materiales de aprendizaje y una herramienta para un aprendizaje efectivo.

Otra de las inquietudes analizadas en varias publicaciones son los factores identificados como importantes para la implementación con éxito de la metodología m-learning en las universidades (Almaiah y Man, 2016; Estrada y Boude, 2018 y Hernández y Pérez, 2016). Entre los más citados se encuentra la capacitación docente, necesaria para cumplir con su rol de guía integrando su formación pedagógica y tecnológica para dar respuesta a las necesidades del alumnado (GarcíaMartínez et al. 2019). Con relación a los factores que dificultan el uso de los Smartphones como herramienta educativa en el aula encontramos mayoritariamente la falta de gestión administrativa que depende de las políticas de los centros y las deficiencias en las infraestructuras de conectividad (Estrada y Boude, 2018).

El incremento de las investigaciones que identifican el m-learning como objeto de estudio evidenciado en algunas revisiones sistemáticas (García-Martínez et al., 2019 y Lai, 2019) demuestran la creciente confianza depositada en los Smartphones como herramienta educativa. Numerosas instituciones experimentan y analizan los efectos de esta metodología en el rendimiento de sus alumnos. En consecuencia, el presente trabajo pretende aunar los resultados de esas investigaciones con el fin de determinar el efecto en el rendimiento de los alumnos.

\section{Objetivos}

El principal objetivo de este trabajo es evaluar la eficacia de los programas educativos basados en la utilización de smartphones como principal herramienta educativa como medio para mejorar el rendimiento académico en el ámbito universitario.

De este objetivo general se derivan los siguientes específicos:

- Valorar el efecto en el rendimiento académico de la utilización de smartphones como herramienta educativa en educación superior.

- Analizar la posible relación entre los resultados obtenidos y el tiempo de duración de los programas. 
- Examinar las posibles relaciones entre los distintos ámbitos de estudio y la eficacia de la metodología estudiada a través de los datos registrados.

\section{Metodología}

La presente investigación se llevó a cabo considerando las fases recomendadas por Meca (2010) para le realización de este tipo de estudios -1. Formulación del problema, 2. Búsqueda de estudios, 3. Codificación de estudios, 4. Cálculo del tamaño del efecto, 5. Análisis estadístico y 6. Publicación del meta-análisis-. Según dicho autor, los meta-análisis posibilitan el análisis crítico y la síntesis de numerosos trabajos integrando sus resultados a través de un índice de cuantitativo calculado a partir de técnicas estadísticas. Con la pretensión de alcanzar conclusiones válidas y objetivas sobre qué es lo que dicen las evidencias presentadas en distintas investigaciones, acerca de la implicación de los smartphones como herramienta educativa en el rendimiento académico, se realizó una revisión sistemática de la biografía publicada en las bases de datos Scopus, WoS, Eric y Dialnet, seleccionadas bajo criterios relacionados con el impacto y prestigio de las mismas lo que nos lleva a confiar que la calidad y rigurosidad de los estudios que configuran la muestra de este análisis sea aceptable. Los criterios de admisión o exclusión seguidos para la configuración de la muestra analizada estuvieron basados en los estándares de calidad de PRISMA (Urrútia y Bonfill, 2010).

Previo al proceso de búsqueda, se realizó una revisión bibliográfica sobre el m-learning para determinar las palabras claves que constituirían la matriz de búsqueda. Como resultado, fueron incluidos los siguientes términos como imprescindibles para la realización de esta etapa de la investigación: m-learning, mobile learning, aprendizaje móvil y BYOD (Bring Your Own Device). Además, se incluyeron limitaciones como; el tiempo, estudios publicados desde 2016 hasta 2020; el tipo de documento, artículos de revistas científicas; el nivel de académico, todos debían centrarse en el nivel universitario; y el estado, todos los artículos debían ser de acceso libre. La heterogeneidad en el número de respuestas de cada base de datos (Scopus $=1223$, Eric $=542$, Dialnet $=342$ y WoS=179) estuvo motivada, en parte, por las distintas posibilidades de limitación que ofrece cada una. 
En adición a las anteriores premisas se elaboró una lista de condiciones que todos los artículos debían cumplir para ser incluidos dentro del estudio. Los trabajos debían ser estudios experimentales, experimentos o cuasiexperimentos, en los que se midiese el efecto de la utilización de los Smartphones como herramienta educativa en el rendimiento académico de alumnos universitarios y se contase con grupo experimental y de control. En cuanto a los datos, los autores debían incluir en su publicación la media y desviación típica obtenida en sus mediciones, datos necesarios para la tipificación del tamaño del efecto. Aquellos estudios obtenidos como respuesta en dos o más bases de datos fueron eliminados de la lista de aquellas en las que se encontraron en segundo, tercer o cuarto lugar cronológicamente hablando. De tal forma que las investigaciones recopiladas en Scopus que aparecieron en cualquiera de las otras bases fueron atribuidos a la búsqueda de Scopus y así sucesivamente, y en este orden, en relación con las bases Eric, Dialnet y WoS. Teniendo en cuenta el objetivo del estudio, el diseño (dos grupos) y la naturaleza de las variables medidas (continua) se ha optado por la elección de un índice de la familia $d$, que consiste en multiplicar el factor de correlación para muestras pequeñas $(c(m))$ por la diferencia entre las medias de los dos grupos $(y T-, y C)$ y dividirla por la desviación típica conjunta de ambos grupos $(S)$ (Meca, 2010) tal y como se muestra en la siguiente fórmula;(Meca, 2010).

Para comprobar la significación del análisis y su heterogeneidad se utilizó el programa Review Manager 5.3 y se realizó con SPSS una prueba de ANOVA para comprobar la influencia de las variables tiempo y ámbito de estudio en la magnitud del efecto del rendimiento académico.

En la Tabla 1 quedan resumidos los criterios de admisión y exclusión seguidos para la configuración de la muestra.

\section{Tabla 1}

Criterios de admisión y exclusión de estudios

\begin{tabular}{ll}
\hline Criterios de Admisión & Criterios de exclusión \\
\hline Artículos científicos recogidos en las & Artículos no académicos, tesis docto- \\
bases de datos Scopus, WoS, Eric y & $\begin{array}{l}\text { rales y otro tipo de documentos o ar- } \\
\text { tículos no recogidos en las bases de } \\
\text { dialnet. }\end{array}$ \\
\hline datos Scopus, WoS, Eric y Dialnet.
\end{tabular}

Artículos publicados los años 2016, Otros años de publicación. $2017,2018,2019$ y 2020 


\begin{tabular}{ll}
\hline Criterios de Admisión & Criterios de exclusión \\
\hline Artículos de libre acceso & $\begin{array}{l}\text { Artículos de acceso restringido o de } \\
\text { pago. }\end{array}$ \\
\hline $\begin{array}{l}\text { Estudios centrados en el análisis } \\
\text { del efecto de la utilización de los }\end{array}$ & $\begin{array}{l}\text { Educatios centrados en otros niveles } \\
\text { Smartphones como herramienta edu- }\end{array}$ \\
$\begin{array}{l}\text { cativa en niveles universitarios de ca- } \\
\text { rácter presencial. }\end{array}$ & \\
\hline
\end{tabular}

Estudios experimentales o cuasiexpe- Estudios basados en datos cualitativos. rimentales

Estudios de dos grupos (control y ex- Estudios sin grupo de control. perimental).

Artículos que incluyan la Media y la Artículos que publican otras medidas Desviación Estándar del postest de (frecuencias, ganancias etc....). ambos grupos.

Fuente: Elaboración propia.

\section{Resultados}

\section{Variables sustantivas}

Dos variables sustantivas han sido analizadas durante el proceso.

En primer lugar, la distribución geográfica de las investigaciones realizadas sobre la utilización de Smartphones como herramienta educativa incluidas en este estudio y, por tanto, que cumplen las características anteriormente expuestas. Tal y como se muestra en la Figura 1, en primer lugar, se encuentran España, Irán y Taiwán, con cuatro artículos cada uno (el 16\%), componiendo el $48 \%$ de los estudios analizados. En segundo lugar, se encuentran China y Kuwait con dos estudios cada uno (el 7\%). El resto de los países (Arabia Saudí, EE.UU., Egipto, Hungría, Indonesia, Nigeria, Noruega, Países Bajos y Turquía han estado representados por una única investigación (el 4\%). 


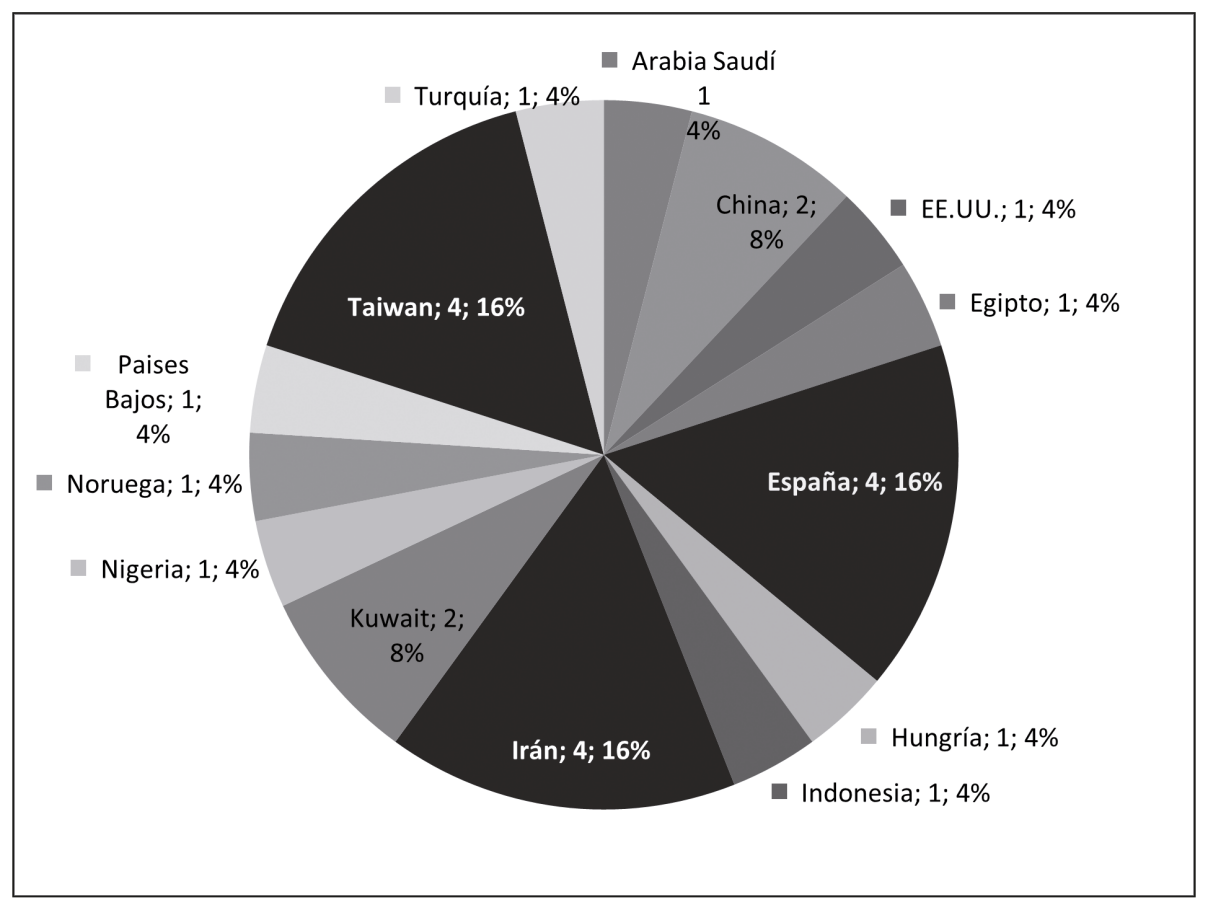

Figura 1. Distribución geográfica de los artículos. Fuente: Elaboración propia.

En segundo lugar, las ramas de conocimientos en las que se han realizado las distintas investigaciones sobre el m-learning mediado por Smartphones en educación presencial universitaria. Con relación a esta, observamos una clara diferencia entre los estudios centrados en el aprendizaje de idiomas, el 52\%, y el resto de los ámbitos. La Figura 2 informa visualmente de la importancia de esta diferencia. 


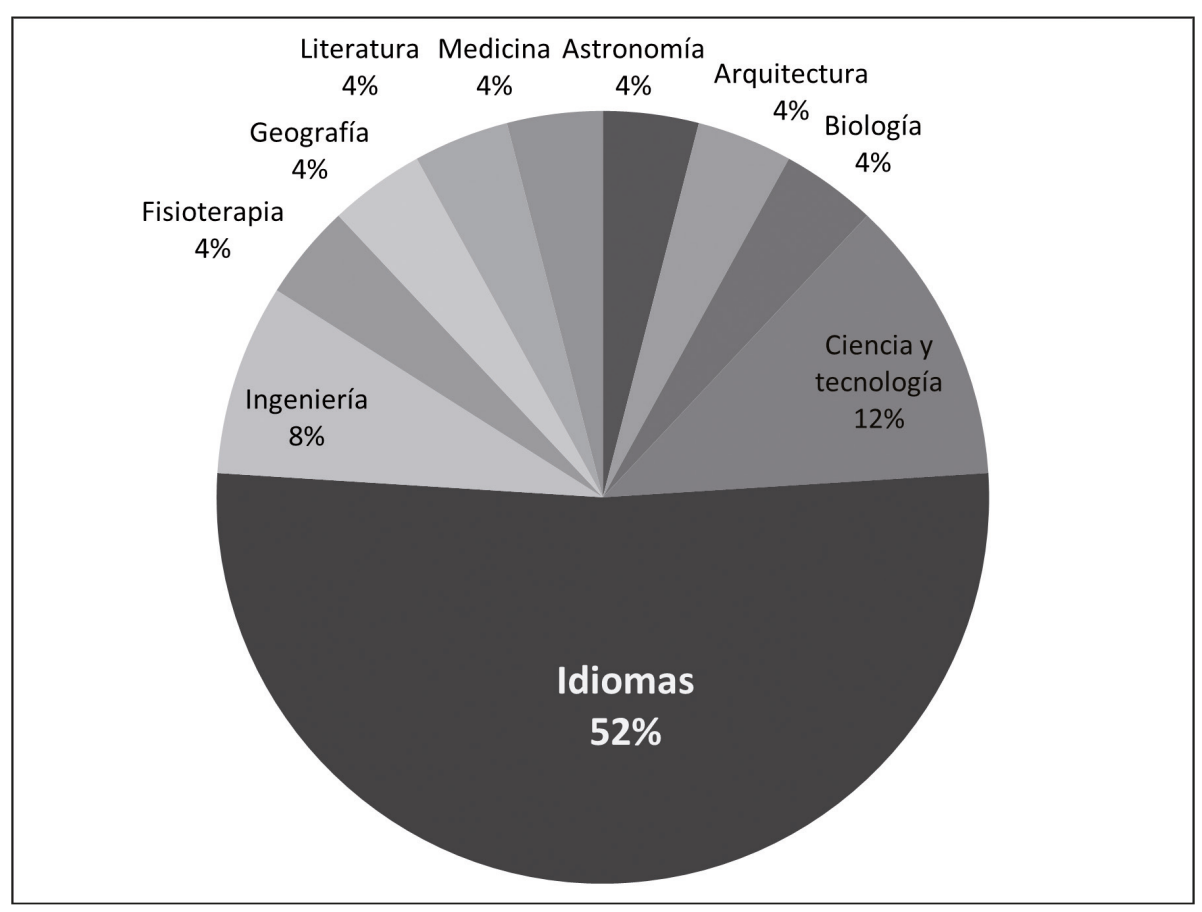

Figura 2. Ámbitos de estudio en los que se centran los artículos. Fuente: Elaboración propia.

Pese a la variabilidad detectada en el resto de estudios; Astronomía, Arquitectura, Biología, Ciencia y tecnología, Idiomas, Ingeniería, Fisioterapia, Geografía, Licenciatura, Literatura y Medicina, se detecta escasa presencia de investigaciones realizadas en ámbitos de Ciencias Sociales o Humanidades. En la Figura 3 se evidencia la distancia entre la cantidad de unos y otros tipos de estudios. 


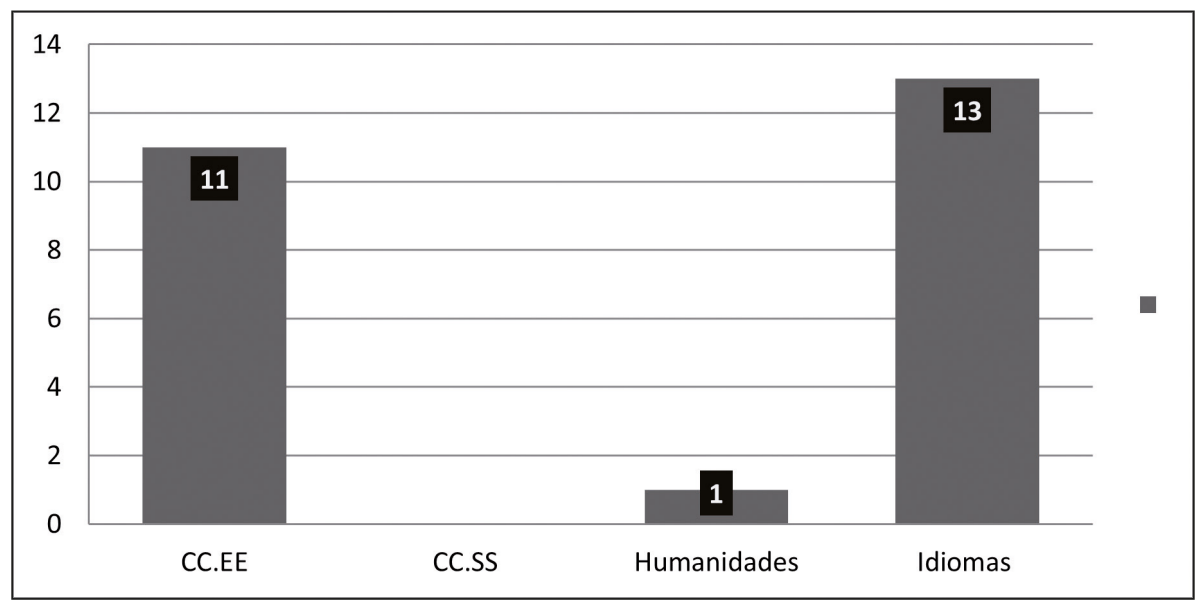

Figura 3. Ramas de conocimiento. Fuente: Elaboración propia.

\section{Variables metodológicas}

Tras la revisión de los estudios obtenidos como respuesta a la búsqueda compuesta por las palabras clave seleccionadas, 2286 en total, pasado el filtro de las premisas preestablecidas y eliminados los artículos duplicados, queda constituida la muestra final $(\mathrm{N}=25)$ para el análisis, 11 artículos obtenidos de Scopus, 9 de Eric, 3 de Dialnet y 2 de WoS.

En relación con el diseño, como puede verse en la Figura 4. el 56\% de los artículos (14) respondieron a las características propias de un diseño cuasiexperimental y el $44 \%$ a uno experimental (11). La mayoría de los estudios realizaron medidas antes y después del tratamiento (21), los cuatro restantes realizaron mediciones sólo después de la intervención. 


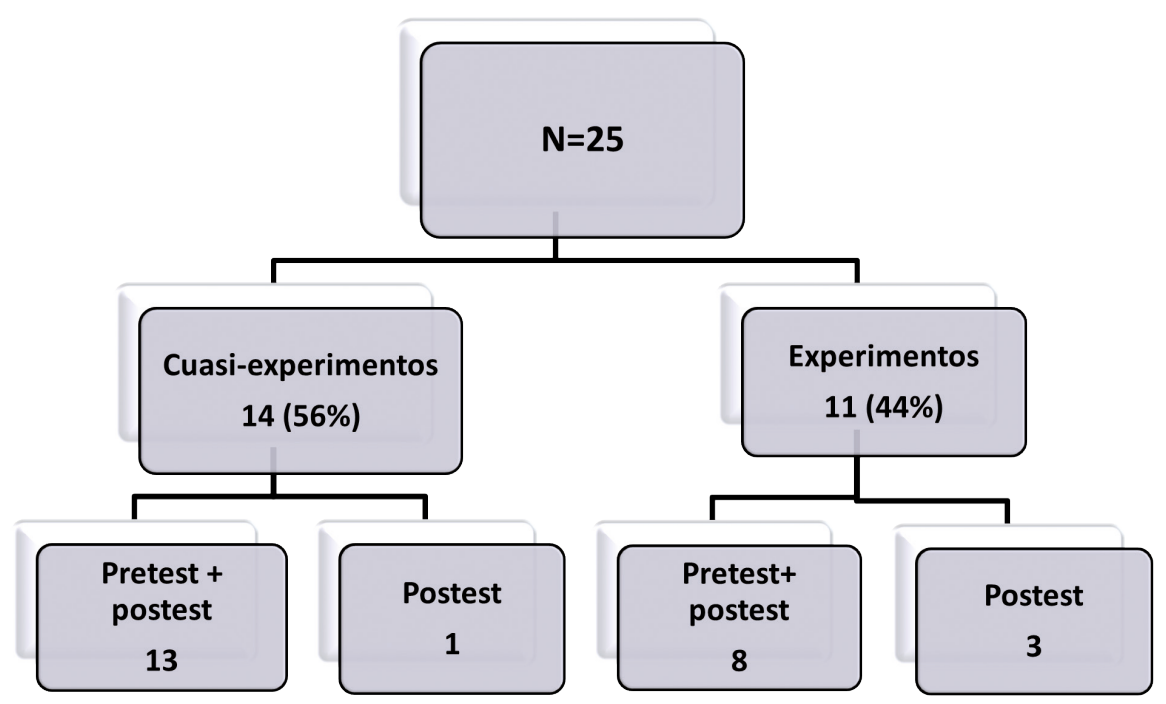

Figura 4. Distribución de los artículos en función de sus características. Fuente: Elaboración propia.

En cuanto a los instrumentos de medida utilizados en los artículos el $52 \%$ (13) optaron por la utilización de cuestionarios, el 16\% (4) realizaron test de opción múltiple, el 20\% (5) eligieron una prueba objetiva de opción múltiple y el 12\% (3) usaron rúbricas para calificar la calidad de los trabajos y/o el nivel de adquisición de conocimiento del alumnado.

El tamaño muestral de cada estudio oscila entre los 25 y 142 participantes $(M=76,32$ y $S=29,62)$ y el tiempo de experimentación varía de entre una sesión a un curso completo (240 días) ( $M=97,92, S=63,67)$. La gran variabilidad detectada en el tiempo de exposición a la metodología juzgada puede considerarse una variable moderadora en el rendimiento del alumnado y que en la magnitud del efecto calculado por lo que más adelante se estudiará la significación de su influencia.

En la siguiente tabla (Tabla 2) se resumen las variables metodológicas y sustantivas relativas a los estudios analizados para este meta-análisis. 
Uxach Molina, B. (2021). Meta-análisis del efecto en el rendimiento académico de los Smartphones como herramienta educativa en educación superior (2016-2020). Educatio Siglo XXI, 39(3),

Tabla 2

Resumen las variables metodológicas y sustantivas relativas a los estudios analizados

\begin{tabular}{|c|c|c|c|c|c|c|}
\hline Autor y año & $\mathrm{N}$ & País & Rama & $\begin{array}{c}\text { Duración } \\
\text { (días) }\end{array}$ & Diseño & Instrumento \\
\hline $\begin{array}{c}\text { Aghajani, et al. } \\
\text { (2018) }\end{array}$ & $\mathrm{N}=70$ & Irán & Idiol & 240 & $\begin{array}{c}\text { Experimen- Pretest y } \\
\text { tal Postest }\end{array}$ & C \\
\hline $\begin{array}{c}\text { Alkhezzi, et al. } \\
(2016) \\
\end{array}$ & $\mathrm{N}=40$ & Kuwait & Idiomas & 84 & $\begin{array}{l}\text { Experimen- Postest } \\
\text { tal }\end{array}$ & $\begin{array}{c}\text { Prueba } \\
\text { objetiva }\end{array}$ \\
\hline $\begin{array}{c}\text { Álvarez, et al. } \\
\text { (2017) }\end{array}$ & $\mathrm{N}=73$ & España & Idiomas & 120 & $\begin{array}{cc}\text { Cuasi-ex- } & \text { Pretest y } \\
\text { perimental } & \text { Postest }\end{array}$ & \\
\hline $\begin{array}{c}\text { Alzubi, y Singh, } \\
\text { (2019) }\end{array}$ & $\mathrm{N}=70$ & $\begin{array}{l}\text { Arabia } \\
\text { Saudí }\end{array}$ & Idiomas & 84 & $\begin{array}{c}\text { Cuasi-ex- Pretest y } \\
\text { perimental Postest }\end{array}$ & \\
\hline Arani, (2016) & $\mathrm{N}=42$ & Irán & Idiomas & 119 & $\begin{array}{c}\text { Cuasi-ex- pretest y } \\
\text { perimental postest }\end{array}$ & \\
\hline Awada, (2016 & $\mathrm{N}=52$ & EE.UU & Idiomas & 28 & $\begin{array}{c}\text { Experimen- pretest y } \\
\text { tal postest }\end{array}$ & ario \\
\hline $\begin{array}{c}\text { Cahyono, et al. } \\
(2019)\end{array}$ & $\mathrm{N}=61$ & $\begin{array}{l}\text { Indone- } \\
\text { sia }\end{array}$ & Idio & 240 & $\begin{array}{c}\text { Cuasi-ex- Pretest y } \\
\text { perimental Postest }\end{array}$ & Rúbrica \\
\hline Chang, & $N=116$ & Taiwán & Ingeniería & 63 & $\begin{array}{c}\text { Cuasi-ex- Pretest y } \\
\text { perimental Postest }\end{array}$ & Rúbrica \\
\hline $\begin{array}{c}\text { Chang, et al. } \\
\text { (2017) }\end{array}$ & $\mathrm{N}=62$ & Taiwán & Ingeniería & 49 & $\begin{array}{c}\text { Cuasi-ex- Pretest y } \\
\text { perimental Postest }\end{array}$ & Rí \\
\hline $\begin{array}{l}\text { Chung, et al. } \\
\text { (2017) }\end{array}$ & $\mathrm{N}=119$ & Taiwán & $\begin{array}{l}\text { Ciencia y } \\
\text { Tecnología }\end{array}$ & 105 & $\begin{array}{c}\text { Cuasi-ex- Pretest y } \\
\text { perimental Postest }\end{array}$ & $\mathrm{Cu}$ \\
\hline $\begin{array}{l}\text { Dai, et al. } \\
(2018) \\
\end{array}$ & $\mathrm{N}=97$ & China & Literatura & 84 & $\begin{array}{c}\text { Experimen- Pretest y } \\
\text { tal postest }\end{array}$ & Cues \\
\hline $\begin{array}{l}\text { Elfeky, et al. } \\
\text { (2016) }\end{array}$ & $\mathrm{N}=50$ & Egipto & Idiomas & 98 & $\begin{array}{c}\text { Cuasi-ex- Pretest y } \\
\text { perimental postest }\end{array}$ & $\begin{array}{c}\text { Prueba } \\
\text { objetiva }\end{array}$ \\
\hline $\begin{array}{c}\text { Fransen, et al. } \\
(2018) \\
\end{array}$ & $\mathrm{N}=62$ & $\begin{array}{l}\text { Países } \\
\text { Bajos }\end{array}$ & Medicina & 42 & $\begin{array}{c}\text { Experimen- Pretest y } \\
\text { tal Postest }\end{array}$ & $\begin{array}{l}\text { Prueba } \\
\text { objetiva }\end{array}$ \\
\hline $\begin{array}{c}\text { Jeno, et al. } \\
(2019)\end{array}$ & $\mathrm{N}=58$ & Noruega & Biología & 7 & $\begin{array}{l}\text { Experimen- Postest } \\
\text { tal }\end{array}$ & Cuestionario \\
\hline Kétyi, (2016) & $\mathrm{N}=94$ & Hungría & Idiomas & 90 & $\begin{array}{c}\text { Cuasi-ex- Pretest y } \\
\text { perimental Postest }\end{array}$ & ario \\
\hline $\begin{array}{c}\text { Lozano- } \\
\text { Lozano, et al. } \\
(2020)\end{array}$ & $\mathrm{N}=105$ & España & $\begin{array}{l}\text { Fisiotera- } \\
\text { pia }\end{array}$ & 14 & $\begin{array}{l}\text { Experimen- Postest } \\
\text { tal }\end{array}$ & Cuestionario \\
\hline $\begin{array}{c}\text { Naderi, N., } \\
\text { y Akrami, A. } \\
(2018)\end{array}$ & $\mathrm{N}=103$ & Irán & Idiomas & 98 & $\begin{array}{cc}\text { Cuasi-ex- } & \text { Pretest y } \\
\text { perimental } & \text { Postest }\end{array}$ & $\begin{array}{l}\text { Prueba } \\
\text { objetiva }\end{array}$ \\
\hline $\begin{array}{c}\text { Oyelere, et al. } \\
(2018)\end{array}$ & $N=142$ & Nigeria & $\begin{array}{l}\text { Ciencias y } \\
\text { Tecnología }\end{array}$ & 91 & $\begin{array}{c}\text { Experimen- Pretest y } \\
\text { tal postest }\end{array}$ & Cuestionario \\
\hline $\begin{array}{l}\text { Redondo et al. } \\
\text { (2017) }\end{array}$ & $\mathrm{N}=25$ & España & $\begin{array}{l}\text { Arquitec- } \\
\text { tura }\end{array}$ & 120 & $\begin{array}{c}\text { Cuasi-ex- Pretest y } \\
\text { perimental Postest }\end{array}$ & Cuestionario \\
\hline
\end{tabular}


Uxach Molina, B. (2021). Meta-análisis del efecto en el rendimiento académico de los Smartphones como herramienta educativa en educación superior (2016-2020). Educatio Siglo XXI, 39(3), 209-234.

\begin{tabular}{|c|c|c|c|c|c|c|}
\hline Autor y año & $\mathrm{N}$ & País & Rama & $\begin{array}{l}\text { Duración } \\
\text { (días) }\end{array}$ & Diseño & Instrumento \\
\hline Safar, (2018) & $\mathrm{N}=100$ & Kuwait & $\begin{array}{l}\text { Ciencia y } \\
\text { Tecnología }\end{array}$ & 120 & $\begin{array}{ll}\text { Cuasi-ex- } & \text { Postest } \\
\text { perimental } & \\
\end{array}$ & Test \\
\hline $\begin{array}{c}\text { Tian, et al. } \\
(2019)\end{array}$ & $\mathrm{N}=50$ & China & $\begin{array}{l}\text { Astrono- } \\
\text { mía }\end{array}$ & 1 & $\begin{array}{c}\text { Experimen- } \text { Pretest y } \\
\text { tal Postest }\end{array}$ & Cues \\
\hline $\begin{array}{l}\text { Turan, et al. } \\
\text { (2018 }\end{array}$ & $\mathrm{N}=95$ & Turquía & Geografía & 21 & $\begin{array}{cc}\text { Experimen- } & \text { Pretest y } \\
\text { tal } & \text { Postest }\end{array}$ & Test \\
\hline $\begin{array}{c}\text { Vaca y Martí- } \\
\text { nez, (2017) }\end{array}$ & $\mathrm{N}=80$ & España & Idiomas & 180 & $\begin{array}{c}\text { Cuasi-ex- } \text { Pretest y } \\
\text { perimental }\end{array}$ & Test \\
\hline Yao, (2015) & $\mathrm{N}=102$ & Taiwán & Idiomas & 90 & $\begin{array}{c}\text { Experimen- } \text { Pretest y } \\
\text { tal postest }\end{array}$ & $\begin{array}{c}\text { Prueba } \\
\text { objetiva }\end{array}$ \\
\hline $\begin{array}{c}\text { Yarahmadzehi } \\
\text { y Goodarzi, } \\
(2020)\end{array}$ & $\mathrm{N}=40$ & Irán & Idiomas & 10 & $\begin{array}{cc}\text { Cuasi-ex- } & \text { Pretest y } \\
\text { perimental } & \text { Postest }\end{array}$ & Test \\
\hline
\end{tabular}

Fuente: Elaboración propia. A partir de los datos obtenidos de los artículos incluidos en el estudio.

\section{Magnitud del Efecto (ME)}

Para el cálculo de la Magnitud del Efecto (ME) de los estudios se tuvo en cuenta la naturaleza continua de la variable de respuesta y la existencia de dos grupos (control y experimental) por lo que se optó por la d de Cohen, como se ha explicado en el apartado relativo a la metodología. La siguiente gráfica (Figura 5) muestra los resultados de este cálculo en que se puede comprobar que la ME es positiva a favor del grupo experimental en todos ellos evidenciando la existencia de una gran variabilidad. 
Uxach Molina, B. (2021). Meta-análisis del efecto en el rendimiento académico de los Smartphones como herramienta educativa en educación superior (2016-2020). Educatio Siglo XXI, 39(3),

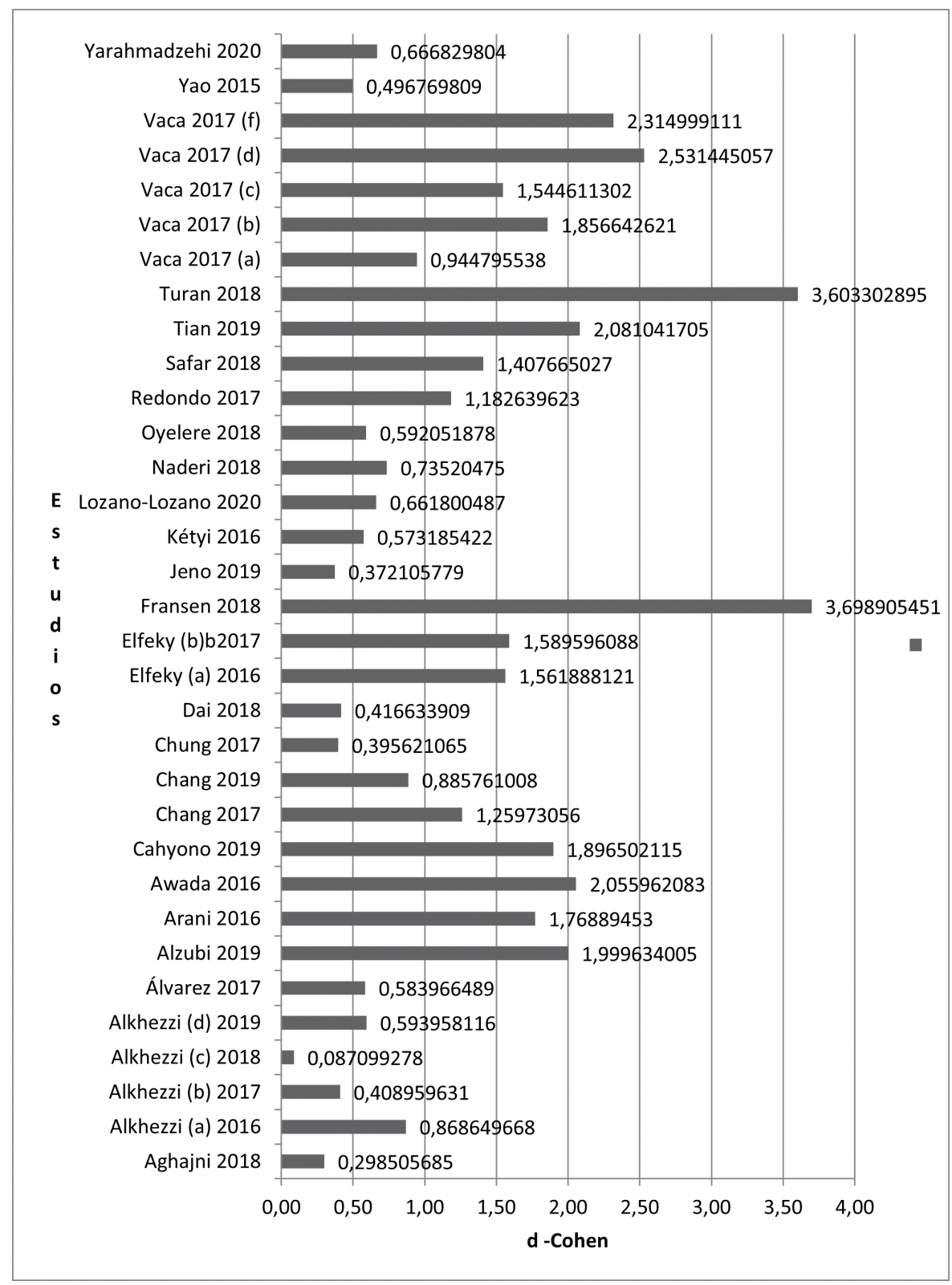

Figura 5. Magnitud del Efecto de m-learning por estudios. Fuente: Elaboración propia. 


\section{Meta-análisis}

Debemos tener en cuenta que pese a que fueron 25 los estudios que configuran nuestra población de estudio, en el análisis estadístico aparecen 33 muestras debido a que tres de ellos proporcionaron más de una medición relativa a distintos aspectos del aprendizaje y todas fueron incluidas. Alkhezzi, et al. (2016), publica mediciones sobre rendimiento en Vocabulario, Gramática, Escritura y General; Elfeky, et al. (2016), en Rendimiento y Conversación y Vaca y Martínez (2017), presentan datos sobre Pronunciación, Gramática, Vocabulario, Fluidez y Comprensión.

Observando el Forest Plot (Figura 6) que apoya nuestro análisis comprobamos que la mayoría de los estudios se encuentran a la derecha de la línea de no efecto, lo que nos informa de que la magnitud del efecto es positiva a favor del grupo que experimento con la metodología basada en la utilización de los Smartphones como herramienta educativa. Aunque los intervalos de confianza de algunas de las muestras cruzan la línea de no efecto, el resultado combinado es significativo ( $Z=9.04$, $p<0.00001)$, como muestra el diamante situado en la parte derecha del gráfico, y a favor del grupo experimental. Por lo que podemos afirmar que en los estudios analizados el m-learning influye positivamente en el rendimiento académico de los alumnos que fueron asignados al grupo experimental.

Concretamente los estudios cuya magnitud del efecto resulta significativa a favor del grupo experimental fueron Aghajani, et al. (2018), Alkhezzi, et al. (a) (2016), Álvarez, et al. (2017), Alzubi, y Singh, (2019), Arani, (2016), Awada, (2016), Cahyono, et al. (2019), Chang, (2019), Dai, et al. (2018), Elfeky, et al. (2016), Fransen, et al. (2018), Kétyi, (2016), Lozano-Lozano, et al. (2020), Naderi, N., y Akrami, A. (2018), Oyelere, et al. (2018), Redondo et al. (2017), Safar, (2018), Tian, et al. (2019), Turan, et al. (2018) y Yarahmadzehi y Goodarzi, (2020) no existiendo estudios a favor del grupo de control. La magnitud del efecto de los estudios Aghajani, et al. (2018), Alkhezzi, et al. (b) (2016), Alkhezzi, et al. (c) (2016), Alkhezzi, et al. (d) (2016), Chang, et al. (2017), Chung, et al. (2017), Jeno, et al. (2019), Vaca y Martínez, (2017), Yao, (2015) resultó no significativa ya que sus intervalos de confianza rozaron o traspasaron la línea de no efecto. 


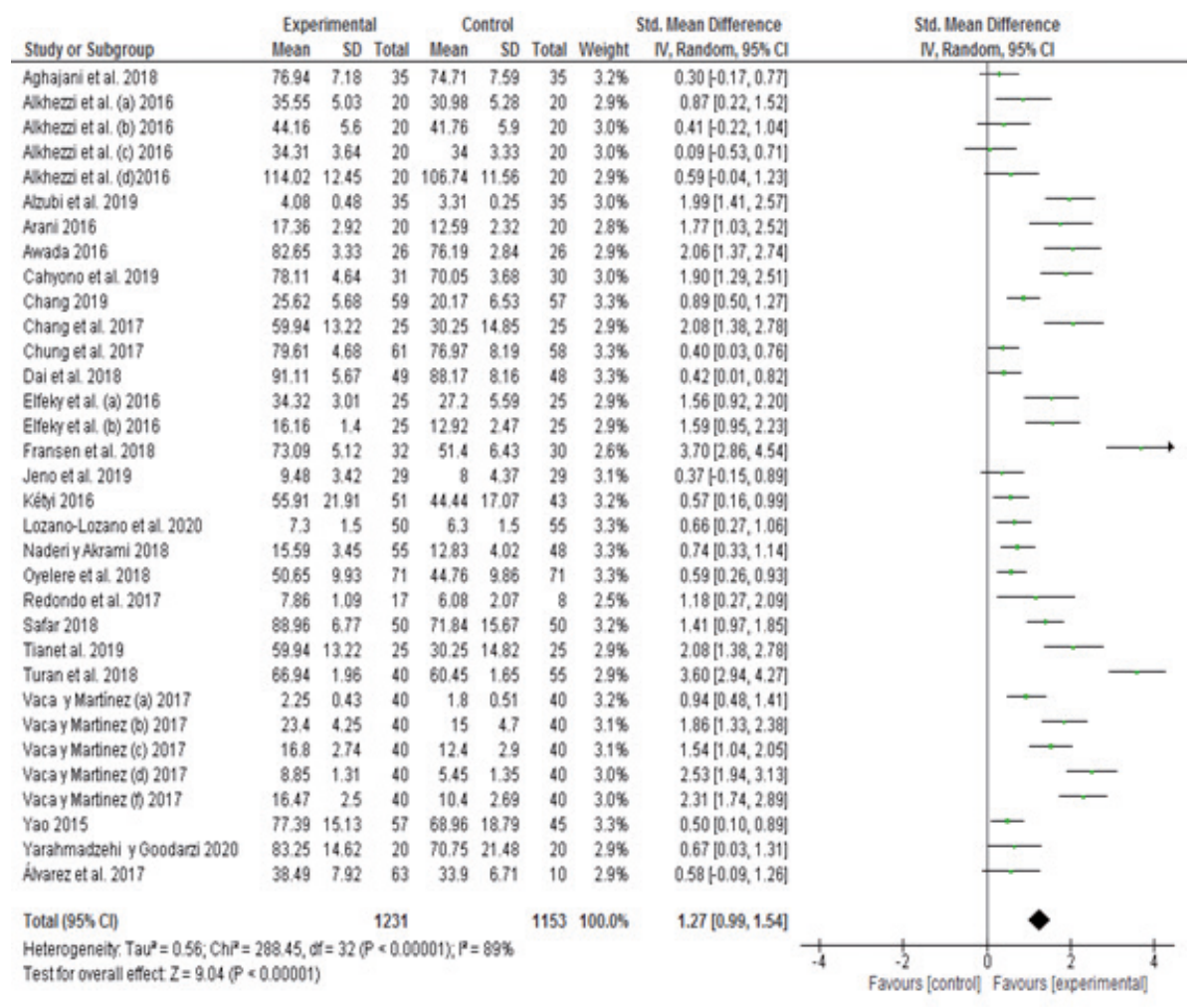

Figura 6. Forest Plot, análisis de la magnitud del efecto conjunto de los artículos. Fuente: Elaboración propia.

Siguiendo con el análisis comprobamos que la heterogeneidad de los estudios es alta, $\mathrm{I}^{2}=89 \%$ lo que nos Ileva a pensar que existe algún otro factor que influye en el rendimiento de los distintos grupos. Tras analizar las diferencias en los diseños de los distintos trabajos se observó que estos diferían en el tiempo de exposición al tratamiento. El tiempo transcurrido entre el comienzo del estudio y la realización del postest osciló entre 1 día y 240, por lo que se creyó oportuno dar respuesta a la siguiente hipótesis: "El tiempo de exposición a la metodología m-learning influye en la magnitud del efecto del rendimiento académico". La prueba de ANOVA (Tabla 3 ) refutó nuestra hipótesis $(f=0.579, p>0.05)$ en la que se concluye que no existen diferencias significativas en la magnitud del efecto del rendimiento académico debidas al tiempo de exposición al tratamiento determinado en cada estudio. 
Uxach Molina, B. (2021). Meta-análisis del efecto en el rendimiento académico de los Smartphones como herramienta educativa en educación superior (2016-2020). Educatio Siglo XXI, 39(3), 209-234.

Tabla 3

Resultado del análisis de ANOVA, relación del tiempo y la magnitud del efecto

\begin{tabular}{|c|c|c|c|c|c|}
\hline \multicolumn{6}{|c|}{ ANOVA de un factor } \\
\hline \multicolumn{6}{|c|}{ Magnitud_efecto } \\
\hline & Suma de cuadrados & $\mathrm{gl}$ & Media cuadrática & $\mathrm{F}$ & Sig. \\
\hline Inter-grupos & ,483 & 1 & 483 &, 579 & 452 \\
\hline Intra-grupos & 25,887 & 31 & 835 & & \\
\hline Total & 26,371 & 32 & & & \\
\hline
\end{tabular}

También se consideró que el m-learning podría influir de forma diferente según el ámbito de estudio sobre el que se experimenta en cada investigación. Por este motivo procedió a dar respuesta a la siguiente hipótesis: "El ámbito de estudio, idiomas u otros, influye en la magnitud del efecto del rendimiento académico mediado por los Smartphones como herramienta educativa". La prueba de ANOVA realizada (Tabla 4) refutó esta hipótesis ( $f=0.266, p<0.05$ ) en la que se concluye que no existen diferencias significativas en la magnitud del efecto del rendimiento académico en función del ámbito de estudio en el que se desarrolló la investigación (idiomas u otros).

Tabla 4

Resultado del análisis de ANOVA, relación del ámbito académico y la magnitud del efecto

\begin{tabular}{llllll}
\hline \multicolumn{1}{l}{ ANOVA de un factor } \\
\hline \multicolumn{2}{l}{ Magnitud_efecto } \\
\hline \multicolumn{1}{l}{ Suma de cuadrados } & gl & Media cuadrática & F & Sig. \\
\hline Inter-grupos &, 224 & 1 &, 224 & $, 266,610$ \\
\hline Intra-grupos & 26,146 & 31 &, 843 & & \\
\hline Total & 26,371 & 32 & & \\
\hline
\end{tabular}

\section{Discusión y conclusiones}

Los datos registrados, tras evaluar la eficacia de los programas educativos basados en la utilización de smartphones como principal herramienta educativa a través de la técnica de meta-análisis, evidencian la pertinencia de implementar la metodología m-learning mediada por Smartphones en el ámbito universitario. Dando respuesta los objetivos planteados en esta investigación, los análisis realizados nos informan 
de que la magnitud conjunta del efecto producido por estas herramientas inteligentes en el aprendizaje es significativa y favorable al grupo experimental. Esta afirmación es independiente del ámbito de conocimiento estudiado y del tiempo de exposición al programa, a pesar de que algunas de las investigaciones analizadas no presentan resultados positivos.

En esta investigación se han analizado dos posibles variables moderadoras, el tiempo de experimentación y el ámbito de estudio, con la intención de dar respuesta a la heterogeneidad de los resultados y a los objetivos planteados. Puesto que ninguna de ellas arroja diferencias significativas en el rendimiento educativo, según los estudios analizados, se cree conveniente para futuras investigaciones analizar otras variables como las aplicaciones utilizadas (Realidad Aumentada, grupos de WhatsApp, aplicaciones específicas...) o el instrumento de evaluación.

En cuanto a las características más reseñables de los estudios analizados observamos mayor producción de estudios en España, Irán y Taiwán que en otros países. Estos estudios se centran de forma mayoritaria en el aprendizaje de idiomas seguido de ámbitos pertenecientes a las Ciencias Experimentales, aunque existe una gran variabilidad. En cuanto al diseño no existe una tendencia clara entre la publicación de estudios cuasi-experimentales y experimentales, aunque como es habitual en Ciencias Sociales se opta en más ocasiones por el primero. Sí se observa que la mayoría de los estudios analizan datos antes y después de la intervención con el fin de eliminar sesgos de agrupación. Y finalmente, en relación con las herramientas de evaluación 13 (52\%) opta por la realización de cuestionarios y el resto se distribuye de manera más equitativa entre pruebas objetivas, test y rúbricas.

Las conclusiones presentadas en este trabajo secundan las averiguaciones de meta-análisis anteriores que examinan desde 2007 estudios similares. Wu et al. (2012) publica un análisis exhaustivo de trabajos publicados entre 2003 y 2010 y Díaz et al. (2018) de editados entre 2007 y 2017. En ambos se ratifica la significación conjunta de las diferencias en el rendimiento entre el grupo control y experimental, siendo estas a favor del último. Por consiguiente, los datos y conclusiones a las que se han Ilegado en este meta-análisis refuerzan los argumentos que confirman la hipótesis planteada en este trabajo: "La utilización de los Smartphones como herramienta educativa mejora el aprendizaje y, por ende, el rendimiento académico". 
La utilización de los Smartphones como herramienta educativa en todos los niveles y en concreto en Educación Superior se ha convertido en un campo de investigación muy interesante debido al gran potencial de estas herramientas en el área. Algunas investigaciones sobre la percepción del profesorado apuntan hacia una cada vez mayor aceptación por el sector, sin embargo, siguen existiendo dudas y temores relacionados con las distracciones del alumnado y la pérdida de control del profesorado (Rodríguez, 2013 y González, y Salcines, 2015). Este tipo de estudios ayuda a la comunidad educativa a disipar las dudas y temores frente a la implementación de la metodología m-learning aportando, de manera conjunta, datos sobre experiencias reales. Puesto que el resultado demuestra una clara influencia positiva en la utilización de los Smartphones como herramienta educativa la tendencia debería verse dirigida hacia una mayor confianza del docente en ella y un aumento en la puesta en práctica de esta metodología ubicua.

Para finalizar y en lo referente al sesgo de publicación hemos de resaltar que este puede estar presente en el meta-análisis que aquí se presenta debido a que en la mayoría de ocasiones las investigaciones con resultados negativos no son publicadas. Por este motivo es difícil indagar sobre los casos o situaciones en los que esta metodología emergente pudiera no ser efectiva y, en caso de que los hubiera, este hecho podría desvirtuar la veracidad de este trabajo.

\section{Referencias}

Aghajani, M., y Adloo, M. (2018). The Effect of Online Cooperative Learning on Students' Writing Skills and Attitudes through Telegram Application. International Journal of Instruction, 11(3), 433-448.

Alkhezzi, F., y Al-Dousari, W. (2016). The Impact of Mobile Learning on ESP Learners' Performance. Journal of Educators Online, 13(2), 73-101.

Almaiah, M. A., y Man, M. (2016). Empirical investigation to explore factors that achieve high quality of mobile learning system based on students' perspectives. Engineering science and technology, an international journal, 19(3), 1314-1320.

Álvarez, I. A., Núñez, M. M., Hernando, A. B. G., y da Silva Fariña, A. (2017). Up2B2: Playing English grammar games at the B2 level. Language Value, (9), 132-159.

Alzubi, A. A. F., y Singh, M. K. A. (2019). Investigating Reading Learning Strategies through Smartphones on Saudi Learners' Psychological Autonomy in Reading Context. International Journal of Instruction, 12(2), 99-114.

Arani, J. A. (2016). Mobile educational SMSs as supplementary means to teach sentence 
Uxach Molina, B. (2021). Meta-análisis del efecto en el rendimiento académico de los Smartphones como herramienta educativa en educación superior (2016-2020). Educatio Siglo XXI, 39(3),

paraphrasing in EMP course. International Journal of Interactive Mobile Technologies (iJIM), 10(1), 45-51.

Awada, G. (2016). Effect of WhatsApp on critique writing proficiency and perceptions toward learning. Cogent Education, 3(1), 1264173.

Brazuelo, F., y Gallego, D. J. (2011). Mobile Learning. Los dispositivos móviles como recurso educativo. Sevilla: Editorial MAD.

Burbules, N. (2012). El aprendizaje ubicuo y el futuro de la enseñanza. Encuentros de Educación. (13), 3-14. Disponible en:

http://library.queensu.ca/ojs/index.php/encounters/article/viewFile/4472/4498. Recuperado el 03/11/19

Cahyono, B. Y., y Astuti, U. P. (2019). Effect of Process Writing Approach Combined with Video-Based Mobile Learning on Indonesian EFL Learners' Writing Skill across Creativity Levels. International Journal of Instruction, 12(3), 325-340.

Chang, Y. S. (2019). The mediating role of motivation for creative performance of cloudbased m-learning. Australasian Journal of Educational Technology, 35(4), 34-45.

Chang, Y. S., Chen, S. Y., Yu, K. C., Chu, Y. H., y Chien, Y. H. (2017). Effects of cloud based $\mathrm{m}$ learning on student creative performance in engineering design. British Journal of Educational Technology, 48(1), 101-112.

Chrifi, I., Tace, E., Radid, M. y Yazza, Y. (2019). Learning electrolysis with podcasting in the higher education: From implementation to results. International Journal of Recent Technology and Engineering, 8(3), 8204-8208.

Chung, C. C., Dzan, W. Y., Cheng, Y. M., y Lou, S. J. (2017). On the push-pull mobile learning of electric welding. Eurasia Journal of Mathematics Science \& Technology Education, 13(7), 3235-3260.

Contreras, R. S. (2010). Percepciones de estudiantes sobre el Aprendizaje móvil; la nueva generación de la educación a distancia. Disponible en:

http://hdl.handle.net/10854/2245. Recuperado el 03/02/2020

Dai, G., Liu, Y., y Cui, S. (2018). A Study on the Mobile Learning of English and American Literature Based on WeChat Public Account. English Language Teaching, 11(6), 47-66.

Díaz, F., y Hernández, G. (2002). Constructivismo y aprendizaje significativo. Estrategias docentes para un aprendizaje significativo: una interpretación constructivista (2a ed.). México: McGraw-Hill. Disponible en:

http://metabase.uaem.mx/handle/123456789/647 Recuperado el 13/11/19

Díaz, I. A., Reche, M. P. C., y Rodríguez, J. M. R. (2018). Efecto de la metodología mobile learning en la enseñanza universitaria: meta-análisis de las investigaciones publicadas en WOS y Scopus. RISTI-Revista Ibérica de Sistemas e Tecnologias de Informação, (30), 1-16.

Elfeky, A. I. M., y Masadeh, T. S. Y. (2016). The Effect of Mobile Learning on Students' Achievement and Conversational Skills. International Journal of higher education, 5(3), 20-31.

Estrada, E. J., y Boude, Ó. R. (2018). Multivariate Analysis of Elements Related to Mobile Learning in Higher Education in Colombia. Revista Electrónica Educare, 22(3), 110128. 
Uxach Molina, B. (2021). Meta-análisis del efecto en el rendimiento académico de los Smartphones como herramienta educativa en educación superior (2016-2020). Educatio Sig/o XXI, 39(3), 209-234.

Fransen, F., Martens, H., Nagtzaam, I., y Heeneman, S. (2018). Use of e-learning in clinical clerkships: effects on acquisition of dermatological knowledge and learning processes. International journal of medical education, 9, 11-17.

García, N. y Álvarez, M. B. (2007). La motivación del alumnado a través de la satisfacción con la asignatura. Efecto sobre el rendimiento. Estudios Sobre Educación, 13, 89-112.

García-Martínez, I., Fernández-Batanero, J. M., Cobos-Sanchiz, D. y Luque de La Rosa, A. (2019). Using Mobile Devices for Improving Learning Outcomes and Teachers' Professionalization. Sustainability, 11(24), 6917.

González-Fernández, N. y Salcines-Talledo, I. (2015). El Smartphone en los procesos de enseñanza-aprendizaje-evaluación en Educación Superior. Percepciones de docentes y estudiantes. RELIEVE. Revista Electrónica de Investigación y Evaluación Educativa, 21(2), 1-20.

Grund, B., Gil, G., y González, C. (2017). Los docentes ante la integración educativa del teléfono móvil en el aula. Revista de Educación a distancia, 17(52).https://www. ine.es/dyngs/INEbase/es/operacion.htm?c=estadistica_C\&cid=1254736176741\&me nu=ultiDatos\&idp=1254735976608 Recuperado el 02/11/2020 https://www.ine.es/ prensa/tich_2019.pdf Recuperado el 02/11/2020

Jabali, O., Saeedi, M., Shbeitah, G., y Ayyoub, A. A. (2019). Medical faculty members' perception of smartphones as an educational tool. BMC medical education, 19(1), 264.

Jeno, L. M., Adachi, P. J., Grytnes, J. A., Vandvik, V., y Deci, E. L. (2019). The effects of $m$ learning on motivation, achievement and well being: A Self Determination. Theory approach. British Journal of Educational Technology, 50(2), 669-683.

Kétyi, A. (2016). From mobile language learning to gamification: An overlook of research results with business management students over a five-year period. Porta Linguarum: revista internacional de didáctica de las lenguas extranjeras, (1), 45-59.

Khan, M. S. H., Abdou, B. O., Kettunen, J., y Gregory, S. (2019). A Phenomenographic Research Study of Students' Conceptions of Mobile Learning: An Example From Higher Education. Sage Open, 9(3), 1-17.

Klimova, B., y Polakova, P. (2020). Students' perceptions of an EFL vocabulary learning mobile application. Education Sciences, 10(2), 37.

Lai, C. L. (2019). Trends of mobile learning: A review of the top 100 highly cited papers. British Journal of Educational Technology, 51(3), 721-742.

López, F.A., Silva, M.M. (2016), Factores que inciden en la aceptación de los dispositivos móviles para el aprendizaje en educación superior, Estudios sobre Educación, 30, 175-195

Lozano-Lozano, M., Galiano-Castillo, N., Fernández-Lao, C., Postigo-Martin, P., ÁlvarezSalvago, F., Arroyo-Morales, M., y Cantarero-Villanueva, I. (2020). The Ecofisio Mobile App for Assessment and Diagnosis Using Ultrasound Imaging for Undergraduate Health Science Students: Multicenter Randomized Controlled Trial. Journal of medical Internet research, 22(3), e16258.

Martín-Gutiérrez, J., Saorín, J. L., Contero, M., Alcañiz, M., Pérez-López, D. C., y Ortega, M. (2010). Design and validation of an augmented book for spatial abilities develop- 
Uxach Molina, B. (2021). Meta-análisis del efecto en el rendimiento académico de los Smartphones como herramienta educativa en educación superior (2016-2020). Educatio Siglo XXI, 39(3),

ment in engineering students. Computers y Graphics, 34(1), 77-91.

Meca, J. S. (2010). Cómo realizar una revisión sistemática y un meta-análisis. Aula abierta, 38(2), 53-64.

Naderi, N., y Akrami, A. (2018). EFL Learners' Reading Comprehension Development through MALL: Telegram Groups in Focus. International Journal of Instruction, 11(2), 339-350.

Oyelere, S. S., Suhonen, J., Wajiga, G. M., y Sutinen, E. (2018). Design, development, and evaluation of a mobile learning application for computing education. Education and Information Technologies, 23(1), 467-495.

Pérez, O. (2009). Diversos condicionantes del fracaso escolar en la educación secundaria. Revista iberoamericana de educación, 51(1), 67-85.

Persson, V., y Nouri, J. (2018). A systematic review of second language learning with mobile technologies. International Journal of Emerging Technologies in Learning (iJET), 13(02), 188-210.

Redondo Domínguez, E., Fonseca Escudero, D., Sánchez Riera, A., y Navarro Delgado, I. (2017). Educating Urban Designersusing Augmented Reality and Mobile Learning Technologies/Formación de Urbanistas usando Realidad Aumentada y Tecnologías de Aprendizaje Móvil. Revista iberoamericana de educación a distancia, 20(2), 141-165.

Rodríguez, M. N. (2013). El m-learning y los usos de tablets y celulares en el aula de clase, ¿distractores o dinamizadores del aprendizaje?. Praxis investigativa ReDIE: revista electrónica de la Red Durango de Investigadores Educativos, 5(8), 94-100.

Safar, A. H. (2018). BYOD in Higher Education: A Case Study of Kuwait University. Journal of Educators Online, 15(2), 1-13.

Santiago, R., y Trabaldo, S. (2015). Mobile learning: nuevas realidades en el aula. México: Editorial Océano.

Tian, K., Urata, M., Endo, M., Mouri, K., Yasuda, T., \& Kato, J. (2019). Real-World Oriented Smartphone AR Supported Learning System Based on Planetarium Contents for Seasonal Constellation Observation. Applied Sciences, 9(17), 3508.

Turan, Z., Meral, E., y Sahin, I. F. (2018). The impact of mobile augmented reality in geography education: achievements, cognitive loads and views of university students. Journal of Geography in Higher Education, 42(3), 427-441.

Urrútia, G., y Bonfill, X. (2010). Declaración PRISMA: una propuesta para mejorar la publicación de revisiones sistemáticas y metaanálisis. Medicina clínica, 135(11), 507511.

Vaca, A. A., y Martínez, M. S. C. (2017). Mensajería instantánea móvil: Whatsapp y su potencial para desarrollar las destrezas orales. Comunicar: Revista científica iberoamericana de comunicación y educación, (50), 43-52.

Wang, Y.-C. Hsu, L. (2020). Shall we go to the MALL? - Students' perceptions of a business English learning app. International Journal of Information and Education Technology, 10(2), 110-116.

Wu, W. H., Wu, Y. C. J., Chen, C. Y., Kao, H. Y., Lin, C. H., y Huang, S. H. (2012). Review of trends from mobile learning studies: A meta-analysis. Computers y Education, 59(2), 817-827.

Yao, C. B. (2015). Constructing a user-friendly and smart ubiquitous personalized lear- 
Uxach Molina, B. (2021). Meta-análisis del efecto en el rendimiento académico de los Smartphones como herramienta educativa en educación superior (2016-2020). Educatio Siglo XXI, 39(3), 209-234.

ning environment by using a context-aware mechanism. IEEE Transactionson Learning Technologies, 10 (1), 104-114.

Yarahmadzehi, N., y Goodarzi, M. (2020). Investigating the Role of Formative Mobile Based Assessment in Vocabulary Learning of Pre-Intermediate EFL Learners in Comparison with Paper Based Assessment. Turkish Online Journal of Distance Education, 21(1), 181-196. 